\title{
The Use of Informal and Formal Help: Four Patterns of Illness Behavior in the Black Community ${ }^{1}$
}

\author{
Harold W. Neighbors ${ }^{2}$ and James S. Jackson \\ Institute for Social Research, The University of Michigan
}

\begin{abstract}
Most studies of professional help use among black Americans fail to describe this group's relationship to blacks experiencing distress but not requesting professional help, and generally ignore the salience of informal social support processes. A more comprehensive understanding of black help-seeking behavior would come from an approach which describes both the users and nonusers of formal helping services, and examines the benefits derived from the interpersonal relationships that comprise black friend-and kin-based networks. These analyses focused on four patterns of informal and formal help use in the National Survey of Black Americans. The findings indicated that most people use informal help only, or they use informal and professional help together. In addition, gender, age, income, and problem-type were significantly related to the different patterns of illness behavior. The implications of these findings for help seeking in the black community were discussed.
\end{abstract}

\footnotetext{
${ }^{1}$ An earlier version of this paper was presented at the 14th annual convention of the Association of Black Psychologists, August 12-15, 1981, Denver, Colorado. The preparation for this manuscript was supported in part by grants from the Ford Foundation, Cénter for Minority Group Mental Health Programs, National Institute of Mental Health, and the National Institute on Aging. We appreciate the comments of Toni Antonucci, Linda Chatters, Gerald Gurin, Rupert Nacoste, Robert Taylor, and three anonymous reviewers on earlier drafts of this paper. Thanks to Lisa Baum, Steve Cardoze, Cathy Jenkins, Jackie Pearlman, Beverly Williams, and Andrea Leibson for their help in the preparation of this manuscript.

${ }^{2}$ All correspondence should be sent to Harold W. Neighbors, University of Michigan, Institute for Social Research, Room 5213, Ann Arbor, Michigan 48106.
} 
Knowledge regarding black help-seeking behavior has been gained secondarily from studies which compare black and white utilization rates of professional health and mental health facilities. These rates provide information only about the characteristics of those blacks who have, by some unspecified means, entered the professional health care system. This focus neglects the fact that there are blacks with serious personal problems who do not seek professional help. What resources do these "nonusers" have at their disposal to ameliorate their problems?

The National Survey of Black Americans, the source of data for the present study, included items to assess whether or not blacks sought professional help for their personal problems. In addition to professional utilization, the survey focused on whether or not respondents sought help from informal social network members. The purpose of this paper was to investigate factors related to informal and professional help seeking in response to stressful life situations among black Americans. Specifically, the following four response patterns were investigated: (a) the use of informal help only, (b) the use of formal help only, (c) the use of both informal and formal help, and (d) the decision not to use any outside help at all.

There have been few attempts to investigate the use of informal social networks for personal problems among blacks. More importantly, previous studies have not examined how blacks use informal and professional help in combination. A more complex description of black help seeking can be provided by investigating informal and professional help use in combination. When the use of professional help is examined in isolation, the implication is that people with problems who do not seek formal assistance go unaided. A recent national study of help-seeking behavior has shown, however, that this is not the case (Veroff, Kulka, \& Douvan, 1981). Given the literature on the importance of informal networks for blacks it is unlikely that those blacks who do not seek professional help go completely without assistance during times of stress.

Informal social networks play an important role in the lives of black people (Hayes \& Mindel, 1973; Martineau, 1977; McAdoo, 1978; Stack, 1974). It is generally accepted that social networks are utilized by blacks as an alternative to formal help seeking as well as a supplement to professional help utilization. It is also known that the structural characteristics of social networks can influence whether or not professional help sources are utilized (Freidson, 1960; McKinlay, 1973; Horwitz, 1977b). Having the ability to empirically document how prevalent the use of both informal and professional help (during the same problem episode) is in the black community among demographically different individuals is important. If a large number of blacks in distress display this particular help-seeking response, it argues strongly for further investigations which focus more specifically on 
what particular aspects of informal social networks (size, density, kindomination, referral-advice) influence the use (or nonuse) of professional resources. On the other hand, if it is found that large numbers of blacks use informal help only, it would necessitate more extensive inquiry into the kinds of assistance offered by different informal helpers for different types of problems.

Despite the fact that most people under stress do seek some form of aid (informal, formal, or both), there are those who choose not to use any external sources of help. Veroff et al. (1981) reported that $13 \%$ of their sample sought neither informal nor formal help during times of crisis. Indeed, we expect to find that there are blacks who do not have access to any helping resources. No matter how small this group may actually be, however, they are an important risk group - in need of help but unwilling or unable to obtain it. If the use of informal and professional help is not viewed in combination, however, this group of true nonusers cannot be identified.

In summary, this article reports on the relationships of sociodemographic (income, age, gender) and personal problem characteristics (type of problem) to four patterns of help seeking. The decision to focus on these particular factors was dictated by previous literature and the expected importance of these social and economic indicators in the help-seeking behavior of black Americans. Previous research in gender differences has clearly implicated its importance in professional help use. Other studies have shown that men and women may differ in the availability and use of informal networks (Horowitz, 1977a). Similarly, previous studies have demonstrated that older age groups may differ from younger age groups in their use of both professional and informal help (Jackson, Neighbors, \& Seaton, 1981). Low family income has been shown to be a potential barrier to the use of formal help (Neighbors, 1984) and as a predictor of high informal help use (McAdoo, 1978). Finally, type of problem is an important control variable in the examination of the relationship of these factors to patterns of help-seeking. In addition, the investigation of how various types of life problems are differentially referred to the informal and formal help system also has important theoretical and practical implications.

\section{METHOD}

\section{Sample}

The analyses to be reported were conducted on a nationally representative cross-section sample of the adult (18 years of age and older) black 
population living in the continental United States. The sample was drawn according to a multistage area probability procedure designed to insure that every black household had the same probability of being selected for the study. This self-weighting sample is unique because never before has a set of procedures been developed that would permit a true probability sample of the entire black population.

Based on the 1970 census (and subsequent updates) distribution of the black population, 76 primary areas were selected. These sites were stratified according to racial composition and income; then smaller geographical areas (clusters) were randomly chosen. Actual sampling and interviewing were conducted in these smaller geographical areas generally representing city blocks or groups of blocks. Preliminary scouting of the selected clusters within each primary area provided a check of new construction, destruction, number of households, and racial composition.

Since correct identification of eligible dwelling units was critical, two special screening procedures were developed for finding black households. The Standard Listing and Screening Procedure (SLASP) which was applied in mixed and mostly black areas provided a unique method of identifying black households by using reference housing units. When all housing units were identified by referents as black-occupied or "other"-occupied, the selection of eligible housing units was taken from the list of black housing units. A subset of the "other"-occupied housing units were selected for screening to assess the accuracy of the informants in the reference housing units. The Wide Area Screening Procedure (WASP) was developed for use in areas with few or no black-occupied housing units. Whereas the SLASP interviewers listed and classified each housing unit in a cluster, the WASP interviewers asked the reference housing unit about blacks in the area and listed only the black-occupied housing units. In order to check the effectiveness of the procedure, $20 \%$ of the WASP clusters were chosen at random and received the more thorough SLASP coverage. The WASP procedure allowed the self-weighting probability sample to be obtained because it permitted, at acceptable cost, the screening of large areas of the country where blacks represented less than $1 \%$ of the population. These blacks had the same probability of selection as blacks who lived in areas that were more heavily black-occupied.

With each selected black household, a single person was randomly chosen to be interviewed. No substitutions were allowed. A refusal to be interviewed by the selected person resulted in the household being classified as a nonresponse. All interviewing was conducted by professionally trained black interviewers. This sample and interviewing procedure resulted in 2,107 completed interviews conducted during 1979 and 1980, representing a response rate of $69 \%$. The black population is disproportionately distributed within urban areas where response rates have traditionally been low. 
The relatively high overall response rate was achieved by intensifying efforts in these urban areas through repeated call-backs.

\section{Instrument}

The section of the questionnaire designed to study help seeking was problem-focused. Respondents were asked about one specific life situation that had caused them a significant amount of distress. If a person had ever experienced a personal problem of this nature they were asked what the problem was about. For analytic purposes, responses were categorized as follows: (a) physical health, (b) interpersonal, (c) emotional, (d) death of a loved one, and (e) economic. In addition, respondents were asked a series of questions designed to elicit information on how they adapted to that personal problem. This paper focuses on two adaptational responses in particular: the seeking of aid from informal and formal help sources. To measure the use of informal help, respondents were presented with a list of lay helpers and asked if they had talked to any of these people about their problem. The informal helper list included spouse, son, daughter, father, mother, brother, sister, other relative, friend, neighbor, and co-worker. Use of help from professionals was measured in a similar manner. Respondents were presented with a list of professional helping facilities and asked if they had gone to any of the places listed for help with their personal problem. The professinal helper list contained the following: hospital emergency room, medical clinic, doctor's office, social service agency, mental health center, private therapist, minister, lawyer, police, school and employment agency.

\section{RESULTS}

Informal help is used more frequently than professional help as a means of coping with problems. Almost half (48.6\%) of those respondents who experienced a problem sought some form of professional help, while the vast majority $\mathbf{8 7 \%}$ ) talked to at least one informal helper. Table I shows that when help seeking is described in terms of the use of informal and professional resources, most people use informal help only $(43 \%)$ or they use both informal and formal help $(44 \%)$. Only $4.3 \%$ enter directly into the professional system without informal consultation. Finally, $8.7 \%$ of those with a serious problem seek no outside assistance at all. ${ }^{3}$

${ }^{3}$ These two help-seeking responses are significantly related to each other $\left(\chi^{2}(1)=17.5\right.$, $p<.001)$. Specifically, half $(569 / 1,124)$ of the people who sought informal help also used professional help while only a third $(56 / 168)$ of those who did not seek informal help sought professional help. 
Table I. Relationship of Gender, Age, and Income to Patterns of Help Seeking

\begin{tabular}{|c|c|c|c|c|c|}
\hline Variable & $\begin{array}{c}\text { Informal } \\
\text { only }\end{array}$ & $\begin{array}{c}\text { Formal } \\
\text { only }\end{array}$ & Both & $\begin{array}{l}\text { No } \\
\text { help }\end{array}$ & $n$ \\
\hline Univariates & 43.0 & 4.3 & 44.0 & 8.7 & 1,292 \\
\hline \multicolumn{6}{|l|}{ Gender $^{a}$} \\
\hline Male & 46.8 & 4.6 & 37.5 & 11.1 & 432 \\
\hline Female & 41.0 & 4.2 & 47.3 & 7.4 & 860 \\
\hline \multicolumn{6}{|l|}{$\mathrm{Age}^{b}$} \\
\hline 18 to 34 & 47.5 & 2.7 & 41.7 & 8.1 & 592 \\
\hline 35 to 54 & 41.2 & 4.5 & 48.6 & 5.7 & 420 \\
\hline $55 \&$ above & 35.4 & 7.6 & 42.6 & 14.4 & 277 \\
\hline \multicolumn{6}{|l|}{ Income $^{c}$} \\
\hline Under $\$ 10,000$ & 40.1 & 4.8 & 45.2 & 9.9 & 566 \\
\hline$\$ 10,000 \&$ above & 48.0 & 2.3 & 43.1 & 6.6 & 573 \\
\hline \multicolumn{6}{|l|}{ Problem-type $^{d}$} \\
\hline Physical & 26.6 & 8.2 & 60.9 & 4.3 & 184 \\
\hline Interpersonal & 44.2 & 2.2 & 46.6 & 6.9 & 491 \\
\hline Emotional & 41.1 & 9.2 & 33.3 & 16.3 & 141 \\
\hline Death & 49.0 & 1.9 & 41.3 & 7.7 & 104 \\
\hline Economic & 48.2 & 4.4 & 41.0 & 6.4 & 251 \\
\hline
\end{tabular}

${ }^{a} \chi^{2}(3)=13.09, p<.01$.

${ }^{b} \chi^{2}(6)=35.06, p<.001$

${ }^{c} \chi^{2}(3)=13.06, p<.01$

${ }^{d} \chi^{2}(12)=67.28, p<.001$.

Table I also shows the relationships between the three selected sociodemographic variables (family income, gender, age) and the four patterns of help seeking. All three of these variables are significantly related to the patterns of help use. More important, however, is the fact that in each demographic subgroup shown, the largest percentage of respondents use informal help only, or they use informal help in combination with professional help. Finally, Table I reveals that type of personal problem also has a significant effect on help-seeking patterns. With the exception of physical problems, respondents tend to seek informal help only, or to use informal help in combination with professional help. Persons experiencing physical problems are the least likely to use informal help only $(27 \%)$.

Despite the fact that the majority of respondents use informal help only or informal and professional help, there are significant relationships between each sociodemographic variable and patterns of help seeking. ${ }^{4}$ These bivariate results are summarized in column 1 of Table II. Other bivariate

\footnotetext{
${ }^{4} \mathrm{~A}$ problem in interpretation arises, however, because the $\chi^{2}$ statistic with greater than 1 degree of freedom does not indicate the precise source of an association. By combining across columns and rows, however, a $\chi^{2}$ statistic can be calculated for each $2 \times 2$ table contained in the larger table and the exact location of the association can be ascertained. This exploratory strategy was used in the present paper and column 1 of Table II presents the results of these analyses. Another method of finding the source of association in an I $\times \mathrm{J}$ table is to compute a $\chi^{2}$ value for each cell within the table using the formula $(0-E)^{2} / E$, where $0=$ the observed
} 
Table II. Summary of Bivariate and Multivariate Analyses of Help-Seeking Patterns ${ }^{a}$

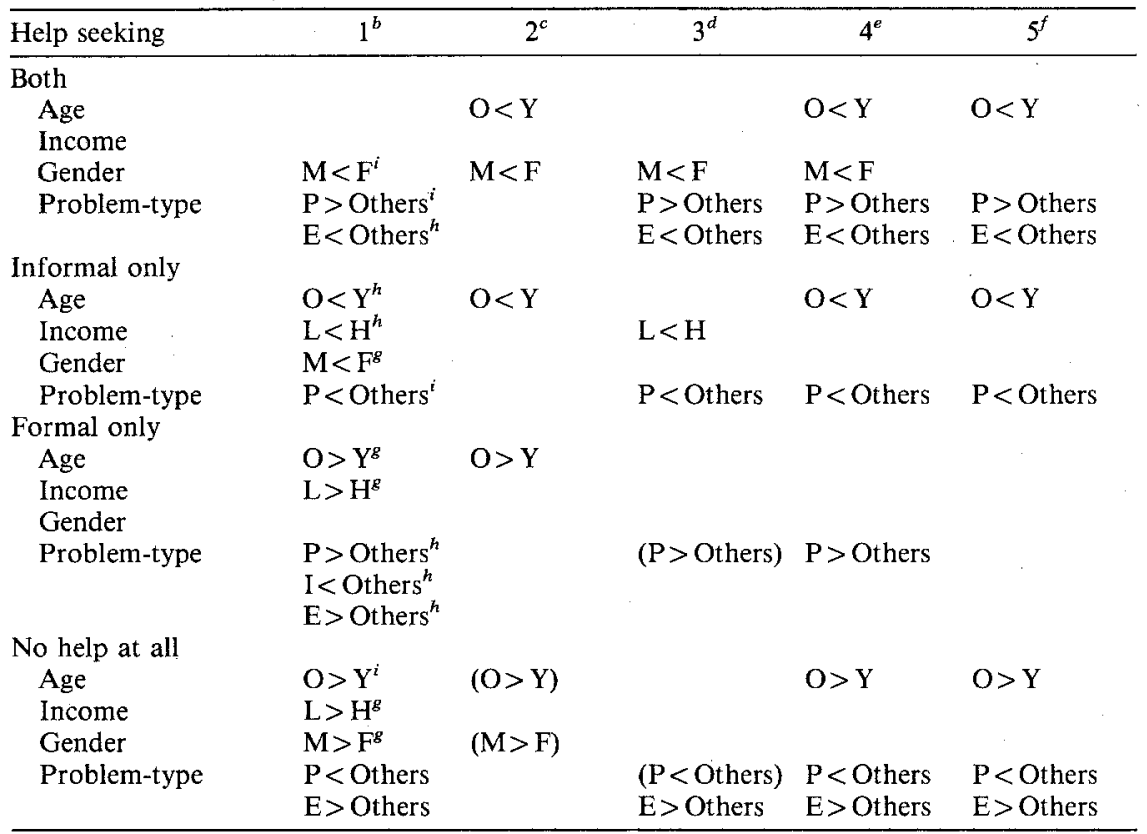

${ }^{a}$ Abbreviations: $\mathrm{M}=$ male, $\mathrm{F}=$ female, $\mathrm{L}=$ low income $(<\$ 10,000), \mathrm{H}=$ high income $(\$ 10,000+), \mathrm{O}=$ older $(55+), \mathrm{Y}=$ younger $(<55), \mathrm{P}=$ physical, $\mathrm{I}=$ interpersonal, $\mathrm{E}=$ emotional. Effects summarized in parentheses approached significance, $\lambda=1.96$.

${ }^{b}$ Bivariate analyses.

${ }^{c}$ Cross-classification of age, income, gender, and use (see Table III).

${ }^{d}$ Cross-classification of problem-type, income, gender, and use (see Table IV).

${ }^{8}$ Cross-classification of problem-type, age, gender, and use (see Table V).

${ }^{f}$ Cross-classification of problem-type, age, income, and use (see Table VI).

${ }^{g} p<.05$.

${ }^{\mathrm{H}} p<.01$.

${ }^{i} p<.001$.

analyses (not shown here) revealed significant relationships between income and age and between income and gender. Both older respondents and women are more likely to be low income. As a result, a multivariate analysis (log-linear models) using income, age, gender, and utilization was conducted. ${ }^{5}$ (See Tables III-VI.) The purpose was to ascertain whether the

frequency and $\mathrm{E}=$ the expected frequency. The cells containing the largest $\chi^{2}$ values give an indication of where the largest contributions to the overall $\chi^{2}$ are located. This analytic strategy was also utilized for each bivariate association of income, gender, age, and problemtype to help-seeking. These results were in agreement with the results obtained by combining the tables.

${ }^{5}$ In the multivariate analysis, the log-linear hierarchical modeling procedure as described by Goodman (1978) and others (Davis, 1974; Knoke \& Burke, 1980) was used. This procedure provides a powerful technique for analyzing categorical data. In this paper a sequential 
Table III. Four-Variable Logit Models for the Cross-Classification of Age (A), Income (I), Gender (G), and Help Seeking $(\mathrm{H})^{a}$

\begin{tabular}{clccc}
\hline Model & Fitted marginals & LR $\chi^{2}$ & $d f$ & $p$ value \\
\hline M1 & $\{\mathrm{H}\}$ & 51.56 & 21 & .000 \\
M2 & $\{\mathrm{HA}\}$ & 30.03 & 18 & .037 \\
M3 & $\{\mathrm{HI}\}$ & 39.02 & 18 & .003 \\
M4 & $\{\mathrm{HG}\}$ & 38.42 & 18 & .003 \\
M5 & $\{\mathrm{HA}\}\{\mathrm{HI}\}$ & 23.73 & 15 & .069 \\
M6 & $\{\mathrm{HA}\}\{\mathrm{HG}\}$ & 16.69 & 15 & .337 \\
M7 & $\{\mathrm{HI}\}\{\mathrm{HG}\}$ & 24.08 & 15 & .064 \\
M8 & $\{\mathrm{HA}\}\{\mathrm{HI}\}\{\mathrm{HG}\}$ & 9.31 & 12 & .676 \\
\hline
\end{tabular}

${ }^{a}$ All models fit (AIG\}.

${ }^{b}$ Preferred model.

bivariate relationships among the demographic variables and patterns of help seeking are affected when the associations among the demographics are taken into account. Column 2 of Table II shows the results of this analysis. Three of the nine relationships observed in the bivariate results are clearly present in this multivariate analysis. Women are more likely than men to use both informal and formal help; older respondents are less likely than the younger group to seek informal help only; older respondents are more likely than younger respondents to seek formal help only. In two instances evidence of a previous significant bivariate relationship being present in the multivariate analysis is less clear. In both cases the value of lambda $(\lambda)$, in relation to its standard error, approaches but does not attain the criterion of significance (1.96) used in this paper. Specifically, there is some evidence that older respondents are more likely than younger people not to seek any help at all. There is also some evidence that men are more likely than women to seek no help. Column 2 also reveals that older respondents are less likely than younger people to use both informal and formal help. This relationship was not present in the bivariate analysis. In four cases relationships observed in the bivariate analysis are eliminated: (a) men are not more likely to seek informal help only; (b) low-income respondents are not more likely to seek professional help only; (c) the low-

modeling process was used to find the most parsimonious logit model which reproduced the observed cell frequencies in a multidimensional contingency table. The maximum likelihoodratio chi-square statistic $\left(\mathrm{LR}_{\chi}{ }^{2}\right)$ is used to assess the goodness of fit between cell frequencies expected under a given model and those actually observed. After a best-fitting model is derived, it is possible to supplement the basic significance tests (derived from the model-fitting process) with additional statistics. In the present analysis the value of $\lambda$ in relation to its standard error was used. Since these coefficients are similar to analyses of variance effects, they can be tested to see if they are significantly different from zero (Veroff et al., 1981, p. 333). Tables III-VI show the log-linear models fit to the various multidimensional contingency tables used in this paper. 
Table IV. Four-Variable Logit Models for the Cross-Classification of Problemtype (P), Income (I), Gender (G), and Help Seeking (H) ${ }^{a}$

\begin{tabular}{clccc}
\hline Model & Fitted marginals & LR $\chi^{2}$ & $d f$ & $p$ value \\
\hline M1 & $\{\mathrm{H}\}$ & 96.06 & 57 & .000 \\
M2 & $\{\mathrm{HP}\}$ & 50.60 & 45 & .262 \\
M3 & $\{\mathrm{HI}\}$ & 86.13 & 54 & .004 \\
M4 & $\{\mathrm{HG}\}$ & 86.82 & 54 & .003 \\
M5 & $\{\mathrm{HP}\}\{\mathrm{HI}\}$ & 42.47 & 42 & .451 \\
M6 & $\{\mathrm{HP}\}\{\mathrm{HG}\}$ & 42.48 & 42 & .450 \\
M7 & $\{\mathrm{HI}\} \mathrm{HG}\}$ & 76.26 & 51 & .013 \\
M8 & $\{\mathrm{HP}\}\{\mathrm{HI}\}[\mathrm{HG}\}$ & 33.67 & 39 & .711 \\
\hline
\end{tabular}

${ }^{a}$ All models fit $\{$ PIG\}.

${ }^{b}$ Preferred model.

income group is not less likely to seek informal help only; and, (d) lowincome people are no longer more likely to seek no help at all.

Another important question is how these relationships between income, age, gender, and help seeking would be affected if type of personal problem was entered into a multivariate analysis. ${ }^{6}$ Columns 3-5 of Table II present the results of these analyses. Analyses which included problem type and gender in the cross-classification (columns 3 and 4) show that women are more likely than men to utilize both informal and formal help. In the two analyses that included age and problem type (columns 4 and 5), older respondents are less likely than younger people to seek both informal and professional help.

For the use of informal help only, in the two analyses which included problem-type and age, it can be seen that older respondents are less likely than younger people to evidence this response (columns 4 and 5, Table II). Of the two analyses which included income, only one shows a significant income effect (column 3 ). In this analysis, the low-income group is less likely than those making $\$ 10,000$ or more to seek informal help only. ${ }^{7}$ (See Table

\footnotetext{
${ }^{6}$ Ideally it would have been preferable to do one five-dimensional analysis (help seeking $x$ income $\times$ age $\times$ gender $\times$ problem-type) but the number of empty cells contained in this five-way cross-classification presents a problem for the log-linear procedure. Another option would have been to do five four-way analyses (i.e., Help seeking $\times$ Income $\times$ Gender $\times$ Age) for each problem-type (physical, interpersonal, emotional, death, economic). This strategy, however, also resulted in an empty cell problem. Therefore, it was decided to do three fourvariable analyses, each containing two of the three demographic factors and all containing the problem-type variable.

'It should be noted that overall, income displays a relatively weak relationship to patterns of help seeking. Neither the preferred model for column 2 (the demographics-only multivariate analysis) nor the preferred model for column 5 (Use $\times$ Problem-type $\times$ Income $\times$ Age) contain an income effect. While the preferred model for the cross-classification of use $x$ Problem-Type $\times$ Income $\times$ Gender (column 3 ) does contain an income effect, it should be pointed out that (a) income and gender result in the same amount of reduction in the $\chi^{2}$ value
} 
Table V. Four-Variable Logit Models for the Cross-Classification of Problemtype (P), Age (A), Gender (G), and Help Seeking (H) ${ }^{a}$

\begin{tabular}{llrrr}
\hline Model & Fitted marginals & LR $\chi^{2}$ & $d f$ & $p$ value \\
\hline M1 & $\{\mathrm{H}\}$ & 141.32 & 57 & .000 \\
M2 & $\{\mathrm{HP}\}$ & 79.07 & 45 & .001 \\
M3 & $\{\mathrm{HA}\}$ & 110.70 & 54 & .000 \\
M4 & $\{\mathrm{HG}\}$ & 131.87 & 54 & .000 \\
M5 & $\{\mathrm{HP}\}\{\mathrm{HA}\}$ & 51.47 & 42 & .150 \\
M6 & $\{\mathrm{HP}\}\{\mathrm{HG}\}$ & 70.54 & 42 & .004 \\
M7 & $\{\mathrm{HA}\}\{\mathrm{HG}\}$ & 101.10 & 51 & .000 \\
M8 & $\{\mathrm{HP}\}\{\mathrm{HA}\}\{\mathrm{HG}\}$ & 42.61 & 39 & .318 \\
\hline
\end{tabular}

${ }^{a}$ All models fit $\{$ PAG $\}$.

${ }^{b}$ Preferred model.

IV.) There is no evidence that men are more likely than women to utilize informal help only in any of the multivariate analyses (a relationship which was present in the bivariate analysis).

Although the bivariate analysis revealed that the low-income group is more likely to seek formal help only, none of the multivariate analyses display evidence of this relationship. While the older group is shown to be more likely to seek formal help only in both column 1 and in column 2 (the multivariate analysis containing demographic variables only), there is no evidence of such an association when problem-type is taken into account.

Finally, the two analyses which include age and problem type (columns 4 and 5) reveal that older people are more likely than younger respondents not to seek any outside help. A $\lambda$ of 1.80 indicated some evidence for this relationship in the cross-classification which included the demographic variables only. The finding that men are more likely than

when compared to the baseline model, $\{\mathrm{H}\}\{\mathrm{PIG}\}$ (see Table IV), (b) the model which fits an effect for problem-type only, $\{$ HP $\}\{$ PIG $\}$ reproduces the observed data very well, $\chi^{2}(45)=$ $50.6, p>.2,3$ ) the two models (M5 and M6, Table IV) which fit the [HP] term along with either $\{\mathrm{HI}\}$ or $\{\mathrm{HG}\}$ fit the observed data equally well, $\chi^{2}(42)=42.5, p>.4,4$ ) both of these models are barely different from the simpler model, [HP]\{PIG\} $\left(\triangle \chi^{2}=8.1, \triangle \mathrm{df}=3\right.$, $p<.05$ ). Thus, while the preferred model for this system does include an income effect, there are indications that the impact of income is not all that great when problem-type is included in the model. These log-linear modeling contrasts (for column 3) also seem to indicate that the relationship between gender and help seeking is not very strong (i.e., note the fact that income and gender resulted in the same amount of reduction in $\chi^{2}$ in comparison to the baseline model). If we look at the series of log-linear models fit to the cross classification of Use $\times$ Problem-Type $\times$ Age $\times$ Gender (see Table V), it can be seen that M8 is the preferred model, but that a simpler model (M5) containing effects for problem-type and age (but not gender) also does an adequate job of reproducing the observed data. The contrast between M5 and M8 is barely significant, however, $\left(\Delta \chi^{2}=8.9, \Delta \mathrm{df}=3, p<.05\right)$. Again, this gives some indication that the gender effect is not all that strong, in comparison to the effects for age and problem-type. 
Table VI. Four-Variable Logit Models for the Cross-Classification of Problemtype (P), Age (A), Income (I), and Help Seeking (H) ${ }^{a}$

\begin{tabular}{clrrc}
\hline Model & Fitted marginals & LR $\chi^{2}$ & $d f$ & $p$ value \\
\hline M1 & $\{\mathrm{H}\}$ & 116.76 & 57 & .000 \\
M2 & $\{\mathrm{HP}\}$ & 71.30 & 45 & .007 \\
M3 & $\{\mathrm{HA}\}$ & 93.12 & 54 & .001 \\
M4 & $\{\mathrm{HI}\}$ & 106.83 & 54 & .000 \\
M5 $^{b}$ & $\{\mathrm{HP}\}\{\mathrm{HA}\}$ & 51.26 & 42 & .155 \\
M6 & $\{\mathrm{HP}\}[\mathrm{HI}\}$ & 63.17 & 42 & .019 \\
M7 & $\{\mathrm{HA}\}\{\mathrm{HI}\}$ & 88.35 & 51 & .001 \\
M8 & $\{\mathrm{HP}\}\{\mathrm{HA}\}\{\mathrm{HI}\}$ & 47.03 & 39 & .177 \\
\hline
\end{tabular}

${ }^{a}$ All models fit $\{\mathrm{PAI}\}$.

${ }^{b}$ Preferred model.

women not to seek help (column 1) is eliminated when problem type is included in the analysis (columns 3 and 4 ).

Next, the relationship of problem-type to utilization (taking the demographic variables into account) is examined. Respondents with physical health problems are more likely than people with other types of problems to use both informal and professional help. This is true for all three of the multivariate analyses containing problem-type, as well as the bivariate analysis (column 1, Table II). Respondents with emotional problems are less likely to use both informal and professional help. This association is also present across all three multivariate analyses including problemtype as well as the bivariate analysis. A consistent pattern for problem-type is also found for the use of informal help only. Here all three multivariate analyses show that people with physical problems are less likely than respondents with other types of problems to utilize informal helpers only. This relationship is also seen in the bivariate results (column 1).

The relationship of problem-type to the response of seeking no help at all is less consistent than the associations just reported. Persons with emotional problems are clearly less likely than respondents with other types of problems not to seek any help. In two of the three multivariate analyses (columns 4 and 5), however, individuals with physical problems are less likely to receive no help at all. This relationship is not present in the bivariate analysis, although it is close to standard significance levels $(p<$ .10). It is also close but not statistically significant at the chosen level $(\lambda=$ -1.90 ) in the four-variable analysis which excludes age.

Other less consistent results appear when the relationship between problem-type and the use of formal help only is investigated. In the bivariate analysis, emotional problems are more likely and interpersonal problems are less likely to be referred for professional help only. These relationships do not appear in any of the multivariate analyses which include 
problem-type. Column 1 of Table II also reveals that persons with physical problems are more likely than respondents with other types of problems to seek formal help only. This association appears in only one of the multivariate analyses (column 4 which includes age and gender but excludes income). The value of lambda, however, in one of the other analyses approaches significance $(\lambda=1.91)$.

In summary, the following six relationships are found in all of the bivariate and multivariate analyses: (a) women are more likely than men to seek both informal and professional help; (b) persons with physical health problems are more likely than people with other types of problems to seek both forms of help; (c) respondents with emotional problems are less likely than those with other types of problems to seek both informal and formal help; (d) older respondents are less likely than younger respondents to seek informal help only; (e) people with physical problems are less likely than those with other types of problems to seek informal help only; (f) respondents with emotional problems are more likely than persons with other types of problems to seek no help. There is fairly strong evidence that older respondents are more likely than younger people not to seek any outside assistance for their problems; and, that people with physical health problems are less likely (than those with other types of problems) not to seek any help at all. Finally, there is some indication that men are more likely than women to seek no help. The latter relationship disappears, however, when problem-type is included in the analysis.

\section{DISCUSSION}

Most help-seeking studies that have focused on blacks examine the use of professional help in isolation. There have been few studies that have explored how the social network influences the use of professional services, and even fewer that have examined different patterns of response as this paper has done. By exploring factors related to the use of informal and professional help in combination, the present investigation adds a level of complexity heretofore missing in analyses of black help-seeking behavior. One shortcoming, however, is that the present analysis does not contain information on time-sequencing. It is not known whether that the informal network is used before making contact with professionals. Although this is probably the case for a majority of respondents, social exchanges undoubtedly occur both prior to and following contact with professional helpers.

The fact that no higher order interactions were uncovered by the multivariate analyses indicates that the relationships between types of problems and help seeking are particularly consistent; they are present regardless 
of the demographic categories. The relationships between the demographic variables and patterns of help seeking are equally consistent; they hold across all types of personal problems.

The help-seeking literature documents the fact that women use professional services at higher rates than men. The data presented here indicate that when utilization is decomposed into its various patterns, men and women do not differ in the sole use of formal services; however, they do differ in the use of formal services in combination with the informal network. Women are more likely to use both informal and formal help together. Simply put, women report getting more help than men. Thus, black women appear to be more affiliative and open in taking their problems to both informal and professional helpers. This finding also raises questions about the differences between the informal networks of men and women and how those differences might influence the use of professional help. For example, Horwitz (1977a) found that women talk to many informal acquaintances about their problems, making themselves more accessible to receiving information about the availability of professional help. Men, on the other hand, appeared more isolated and thus more insulated from receiving information about the availability of professional help. If it is the case that the structural characteristics of one's informal network influence the type of help offered, then it might be possible the type of informal assistance offered to black men works to impede access to professional help.

There was some indication in the bivariate analyses that the poor were more likely not to seek any help and less likely to seek informal help only. The multivariate analyses revealed, however, that income was not very strongly related to patterns of help seeking. In fact, the findings seem to show that the relationship between income and help seeking was really due to age. The findings for income and age were in the same direction. Furthermore, whenever age and income were included together in a multivariate analysis, the income effect was eliminated. These results suggest that the bivariate effects of income on help seeking were really due to the fact that a disproportionate number of older blacks have incomes of less than $\$ 10,000$.

Of the three demographic variables investigated, age showed the strongest and most consistent relationship to help-seeking patterns. The findings indicate that older blacks were more likely than the younger age group not to have sought any help at all. This is the only demographic subgroup explored which showed consistent evidence of being nonusers. This is cause for concern because it indicates that a substantial proportion of older blacks may be in need of help but unwilling or unable to find it. This finding highlights the need to investigate barriers (transportation, money, etc.) the black elderly may face in trying to obtain help for their problems. Respondents age 55 and above were less likely to use both informal and professional help. Older blacks may be more socially isolated 
than their younger counterparts due to the shrinking size of informal networks over the life course (Kahn \& Antonucci, 1981). This could account for the fact that older blacks were also less likely to seek informal help only. With a smaller pool of available helpers, elderly blacks may be unable to rely solely on this source of help. There were some indications in the bivariate results that the older group was more likely to use professional help only, although this relationship was eliminated when problem-type was included in the multivariate analyses. Taken together, these findings suggest that older blacks (in comparison to those under age 55) tend to seek professional help or they seek no help at all.

It is interesting to speculate about the type of informal assistance offered to persons with physical health problems. Such respondents present a very clear picture across all of the multivariate analyses: they were less likely to seek informal help only and more likely to seek both informal and professional help. These results indicate that when personal distress is attributed to a physical health problem (in contrast to other types of problems), blacks more readily turn to their informal helpers for advice; and that advice more often than not is to seek professional help. As indicated previously, information regarding the sequencing of contact between informal and formal helpers is not available. Another plausible explanation may be that blacks with physical health problems are more likely than blacks with other types of problems to consult informal helpers following professional contact. Future analyses of the type of help offered by informal network members for physical problems should clarify this issue. If it is the case that most of the informal advice offered for physical health problems is referral information, it would support the assumption that the majority of respondents sought informal help prior to making professional contact.

While there appears to be a high degree of consensus among blacks that informal and professional help should be utilized for physical problems, just the opposite pattern was evident for emotional problems. These respondents were less likely to seek both sources of help and more likely not to seek any help at all. Of all the types of stressful conditions investigated in this study, emotional difficulties come closest to what might be called "mental health" problems. Instead of referring to a specific event (when asked what the problem was about), respondents in this category gave references to disturbances of feelings, emotions, and mood. The data on emotional problems do not allow us to say whether or not such respondents actually meet the clinical criteria of some discrete psychiatric disorder. These findings do suggest, however, that those blacks who focus on affect and mood as critical factors in defining the nature of their distress are (in comparison to blacks with other types of problems) reluctant to seek informal or professional help. 
Perhaps the most important finding of the present study was the large number of respondents who did make contact with social network members about their problem. The majority of respondents were categorized as users of informal help only, or as users of informal help in combination with professional help, even when demographic background and type of personal problem were taken into account. These findings suggest that research on how the social network functions, both as an alternative treatment source and as a referral system, should prove to be a fruitful area of investigation for social scientists interested in the help-seeking behavior of blacks. This paper has presented data on one important aspect of this topic: how the use of informal help corresponds with the use of professional help. What is needed in the future are more in-depth invvestigations of the specific types of problems taken to the social network and how informal helpers attempt to resolve these problems.

The practical impetus for this research on illness behavior and help seeking derives from an interest in improving human service delivery to blacks who might be in need of professional help, but who may not be receiving it. It is important to arrive at some initial conceptions about those stressful conditions for which it is reasonable to have professionals intervene and those for which the natural community support systems seem to do an adequate job. Making this difficult discrimination requires information on more issues than the present article has been able to address. Among the relevant considerations are (a) the clinical characteristics (from the professional's diagnostic perspective) of the health-related condition that a particular help seeker has; (b) the effectiveness of professional intervention techniques in treating that particular condition; (c) the alternatives to professional help that exist and are engaged in by those suffering from that condition; and (d) the effectiveness of those alternative lay treatment (or helping) strategies.

In short, more information is needed about what is and what is not "appropriate" black illness behavior. Greater congruence between a predominantly white, highly technical health care system and the lay medical system, which we believe guides black help-seeking decisions, can only be developed by investigating how various aspects of black culture affect the utilization of health care resources. This is especially important for designing the focus and content of health educational messages should it seem appropriate to attempt the modification of black illness behavior.

\section{REFERENCES}

Adams, B. (1970). Isolation, function and beyond. American kinship in the 1960s. Journal of Marriage and the Family, 32, 575-597. 
Barnes, E. The black community as the source of positive self concept for black children. In R. L. Jones (Ed.), Black psychology. New York: Harper \& Row, 1972.

Billingsley, A. Black families in white America. Englewood Cliffs: Prentice-Hall, 1968.

Davis, J. A. Hierarchical models for significance tests in multivariate contingency tables. In H. L. Costner (Ed.), Sociological methodology, 1973-1974. San Francisco: Jossey-Bass, 1974.

Friedson, E. Client control and medical practice. American Journal of Sociology, 1960, 65, 374-382.

Goodman, L. A. Analyzing qualitative categorical data. Cambridge: Abt, 1978.

Hayes, W., \& Mindel, C. Extended kinship relations in black and white families. Journal of Marriage and the Family, 1973, 35, 51-57.

Hill, R. (1971). Strengths of black families. Washington, D.C.: National Urban League.

Horwitz, A. The pathways into psychiatric treatment: Some differences between men and women. Journal of Health and Social Behavior, 1977, 18, 169-178. (a)

Horwitz, A. Social networks and pathways to psychiatric help seeking. Social Science and Medicine, 1977, 12, 297-304. (b)

Jackson, J., Neighbors, H., \& Seaton, C. Illness behavior and help seeking for personal problems among the black elderly. Paper presented at the annual meeting of the Gerontological Society of America, November 1981.

Kahn, R., \& Antonucci, T. Convoys over the life course: Attachment roles and social support. In P. B. Baltes \& O. G. Brims (Eds.), Life-span development and behavior. New York: Academic Press, 1980.

Knoke, D., \& Burke, P. Long-linear models. Beverly Hills: Sage, 1980.

Martineau, W. Informal social ties among urban black Americans. Journal of Black Studies, $1977,8,83-104$.

McAdoo, H. Impact of upward mobility on the extended black family. Paper presented at the annual Michigan Symposium on Black Psychology, The University of Michigan, Ann Arbor, April 1978.

McKinlay, J. Social networks, lay consultation and help seeking behavior. Social Forces, 1973 , $51,275-292$.

Mechanic, D. The concept of illness behavior. Journal of Chronic Disease, 1962, 15, 189-194.

Neighbors, $\mathbf{H}$. Professional help use among black Americans: Implications for unmet need. American Journal of Community Psychology, 1984, in press.

Neighbors, H., Jackson, J., Bowman, P., \& Gurin, G. Stress, coping and black mental health: Preliminary findings from a national study. Prevention in Human Services, 1983, 2, 4-29.

Stack, C. All our kin: Strategies for survival in the black community. New York: Harper \& Row, 1974.

Veroff, J., Kulka, R., \& Douvan, E. Mental health in America: Patterns of help seeking from 1957 to 1976. New York: Basic Books, 1981. 